\title{
Repeat Gamma Knife radiosurgery versus microvascular decompression following failure of GKRS in trigeminal neuralgia: a systematic review and meta-analysis
}

\author{
Devi Prasad Patra, MD, MCh, Amey Rajan Savardekar, MD, MCh, Rimal Hanif Dossani, MD, \\ Vinayak Narayan, MD, MCh, Nasser Mohammed, MD, MCh, and Anil Nanda, MD, MPH \\ Department of Neurosurgery, LSU Health Shreveport, Shreveport, Louisiana
}

OBJECTIVE Gamma Knife radiosurgery (GKRS) has emerged as a promising treatment modality for patients with classical trigeminal neuralgia (TN); however, considering that almost half of the patients experience post-GKRS failure or lesion recurrence, a repeat treatment is typically necessary. The existing literature does not offer clear evidence to establish which treatment modality, repeat GKRS or microvascular decompression (MVD), is superior. The present study aimed to compare the overall outcome of patients who have undergone either repeat GKRS or MVD after failure of their primary GKRS; the authors do so by conducting a systematic review and meta-analysis of the literature and analysis of data from their own institution.

METHODS The authors conducted a systematic review and meta-analysis of the PubMed, Cochrane Library, Web of Science, and CINAHL databases to identify studies describing patients who underwent either repeat GKRS or MVD after initial failed GKRS for TN. The primary outcomes were complete pain relief (CPR) and adequate pain relief (APR) at 1 year. The secondary outcomes were rate of postoperative facial numbness and the retreatment rate. The pooled data were analyzed with $\mathrm{R}$ software. Bias and heterogeneity were assessed using funnel plots and $\mathrm{I}^{2}$ tests, respectively. A retrospective analysis of a series of patients treated by the authors who underwent repeat GKRS or MVD after postGKRS failure or relapse is presented.

RESULTS A total of 22 studies met the selection criteria and were included for final data retrieval and meta-analysis. The search did not identify any study that had directly compared outcomes between patients who had undergone repeat GKRS versus those who had undergone MVD. Therefore, the authors' final analysis included two groups: studies describing outcome after repeat GKRS $(n=17)$ and studies describing outcome after MVD $(n=5)$. The authors' institutional study was the only study with direct comparison of the two cohorts. The pooled estimates of primary outcomes were APR in $83 \%$ of patients who underwent repeat GKRS and $88 \%$ of those who underwent MVD $(p=0.49)$, and CPR in $46 \%$ of patients who underwent repeat GKRS and $72 \%$ of those who underwent MVD $(p=0.02)$. The pooled estimates of secondary outcomes were facial numbness in $32 \%$ of patients who underwent repeat GKRS and $22 \%$ of those who underwent MVD ( $p=0.11)$; the retreatment rate was $19 \%$ in patients who underwent repeat GKRS and $13 \%$ in those who underwent MVD ( $p=0.74)$. The authors' institutional study included 42 patients (repeat GKRS in 15 and MVD in 27), and the outcomes 1 year after retreatment were APR in 80\% of those who underwent repeat GKRS and $81 \%$ in those who underwent MVD ( $p=1.0)$; CPR was achieved in $47 \%$ of those who underwent repeat GKRS and $44 \%$ in those who underwent MVD $(p=1.0)$. There was no difference in the rate of postoperative facial numbness or retreatment.

CONCLUSIONS The current meta-analysis failed to identify any superiority of one treatment over the other with comparable outcomes in terms of APR, postoperative facial numbness, and retreatment rates. However, MVD was shown to provide a better chance of CPR compared with repeat GKRS.

https://thejns.org/doi/abs/10.3171/2018.5.JNS18583

KEYWORDS repeat Gamma Knife; microvascular decompression; outcome; pain response; systematic review; meta-analysis; stereotactic radiosurgery; trigeminal neuralgia

ABBREVIATIONS APR = adequate pain relief; BNI = Barrow Neurological Institute; CPR = complete pain relief; EGFP = excellent/good/fair/poor; GKRS = Gamma Knife radiosurgery; $\mathrm{MVD}=$ microvascular decompression; $\mathrm{TN}$ = trigeminal neuralgia.

SUBMITTED March 1, 2018. ACCEPTED May 2, 2018.

INCLUDE WHEN CITING Published online October 26, 2018; DOI: 10.3171/2018.5.JNS18583. 
$\mathrm{G}$ AMMA Knife radiosurgery (GKRS) has emerged as a promising treatment modality in classical trigeminal neuralgia (TN) because of its minimally invasive nature, pain response rates comparable to microvascular decompression (MVD), and low risk of perioperative and postoperative morbidity. Although MVD has traditionally been shown to be superior to GKRS in terms of the degree and duration of pain relief, GKRS is a great treatment option in patients who are elderly, poor surgical candidates, and have no clear vascular compression on imaging studies. ${ }^{2,27}$

GKRS has been shown to be more effective when used as a primary treatment rather than a salvage procedure. ${ }^{10,24}$ Therefore, in many centers GKRS is still practiced as the primary treatment based on both patient and physician preference. However, considering that almost half of the patients have recurrent TN following GKRS, a repeat treatment will be necessary in many patients. The existing literature does not offer clear evidence on which treatment modality, GKRS or MVD, is superior in the treatment of recurrent TN in patients first treated with GKRS.

This combined systematic review and meta-analysis is the first in the neurosurgical literature to evaluate which treatment modality, GKRS or MVD, is superior in the treatment of recurrent $\mathrm{TN}$ in patients first treated with GKRS. In addition, the authors have analyzed and presented their own series of patients with a direct comparison of outcomes.

\section{Methods}

\section{Systematic Review and Meta-Analysis}

We performed a systematic review of the literature to identify studies describing outcome in patients with initial failure or recurrence after GKRS who were treated with either MVD or repeat GKRS. A detailed protocol about the inclusion and exclusion criteria, search strategy, and statistical methods was prepared. The PRISMA (Preferred Reporting Items for Systematic Reviews and Meta-Analysis) guidelines were implemented.

\section{Literature Search Strategy}

Electronic searches were performed using PubMed, Cochrane Library, Web of Science, and CINAHL databases. All the published articles and abstracts were searched from their dates of inception to January 2018 for both English and non-English language literature. A detailed independent search was made with the search manager using a combination of the following key terms or MeSH terms: "trigeminal neuralgia," "gamma knife," "radiosurgery," "stereotactic," "microvascular decompression," "surgery," "failure," "repeat," "refractory," "retreatment," "recurrence," and their synonyms. Two investigators (D.P.P., A.R.S.) performed the literature search. A computerized web search was carried out for any unpublished article or abstract using the same keywords. All the titles and abstracts were reviewed after removing the duplicates. Relevant articles were identified and the full texts were reviewed independently by two reviewers (R.H.D., V.N.). A mismatch was clarified by mutual consensus.

\section{Inclusion and Exclusion Criteria}

Studies describing patients who underwent either GKRS or MVD after initial failed GKRS for idiopathic TN were included. Studies describing patients with TN or atypical facial pain arising from tumor or other intracranial lesions were excluded. Individual case reports or small case series describing fewer than 5 patients were excluded. Abstract-only articles and conference proceedings without full-text publications were excluded. Studies describing the same cohort of patients in different publications were identified, and data from the most recent published article were collected. Age, country of origin, and racial factors were not factors for exclusion.

\section{Outcome Measures and Definitions}

The primary outcomes evaluated were complete pain relief (CPR) at 1 year and adequate pain relief (APR) at 1 year. The secondary outcomes were rate of postoperative/ post-GKRS numbness (of any grade) and the retreatment rate. Other information regarding mean follow-up time, median time interval between subsequent treatments, and median dose of GKRS at first and second treatment was collected. CPR was defined as complete pain relief without any medication. APR was defined as adequate control of pain (more than 50\%) with or without medications. The definitions of CPR and APR using different scales as described by different studies have been outlined in Table 1 . The time of assessment of pain relief varied among studies. In several studies that described pain outcomes at different time intervals, the pain responses at 1 year were used in the analysis. In other studies, we referred to the mean follow-up time, and since all of the studies have mean follow-up times exceeding 1 year, we assumed the described pain outcome to be nearly consistent with an outcome of at least 1 year. Postoperative numbness was defined as any new or increased numbness after repeat GKRS or MVD. Bothersome numbness was defined as Barrow Neurological Institute (BNI) numbness scale score of 2 or the equivalent on another scale. A need of further treatment (of any form) after the repeat GKRS or MVD was considered to be retreatment. Corresponding authors of the studies were contacted by email communications for any missing outcome values.

\section{Assessment of Bias and Study Heterogeneity}

All the abstracts and full-text articles, after removing duplicates, were reviewed independently by two reviewers to eliminate selection bias. We did not find any randomized controlled trials on the topic of interest. As all the studies were either retrospective or prospective observational studies, the quality scoring of the included studies was done by two independent reviewers (D.P.P., A.R.S.) according to the review checklist of the Dutch Cochrane Center proposed by MOOSE. Inclusion criteria in all studies were carefully reviewed to identify any treatment bias. The final results were reviewed by the senior reviewer (A.N.). Funnel plots for primary outcomes for both GKRS and MVD were created, and the significance of plot asymmetry was calculated with Eggers test using a mixed-effects meta-regression model. Study heterogeneity for each analysis was calculated using the Cochran $\mathrm{Q}$ and $\mathrm{I}^{2}$ test. 
TABLE 1. Comparison of pain outcome scales used across studies

\begin{tabular}{clll}
\hline Current Analysis & \multicolumn{1}{c}{ BNI Pain Score (modified) } & Marseille Score (modified) & \\
\hline CPR & I: no pain, no meds & I: no pain, no meds & Excellent: no pain, no meds \\
\hline \multirow{2}{*}{ APR } & II: occasional pain, no meds & II: no pain w/ meds & Good: no pain, reduced meds \\
\cline { 2 - 4 } & IIla: no pain w/ meds & III: $\geq 90 \%$ pain relief & \multirow{2}{*}{ Fair: significantly less pain requiring fewer meds } \\
\cline { 2 - 3 } Failure & IIIb: some pain controlled w/ meds & IV: $\geq 50 \%$ pain relief & \\
\hline
\end{tabular}

meds $=$ medications

\section{Statistical Analysis}

We did not identify any study (excluding our own study) that offered a head-to-head comparative analysis of both the treatment arms. Therefore, our meta-analysis concentrated on combining the outcome measures from individual studies to provide a pooled estimate. A metaanalysis of proportions was conducted with R software version 3.4.3 with RStudio using the "metaphor," "meta," and "ggpubr" packages. For the outcome groups that contained proportions that were not symmetrically distributed (values containing less than 0.2 or more than 0.8 ), a logit transformation was applied. A double arcsine transformation was applied for proportions containing 1.0 or 0.0 . As significant heterogeneity was expected among the included studies, the transformed proportions were combined using DerSimonian-Laird random-effects models and subsequently back transformed to get the pooled estimates. To identify the outlier studies that might have influence on effect size, Baujat plots were created for each outcome measure. Screening for externally studentized residuals was done, and studies with absolute values more than 2 were excluded to get an estimate of the effect without outliers. Forest plots were created to get the summary of proportions with both fixed-effect and random-effects models. An independent-sample t-test was conducted to compare the means between the two treatment arms; $\mathrm{p}<$ 0.05 was considered significant.

\section{Retrospective Review of Our Own Series}

We retrospectively analyzed data obtained in our patients who underwent repeat GKRS or MVD after failure or relapse from previous GKRS at our institute between January 2000 and June 2016. We excluded patients who received any other modality of treatment between the two treatments. We also excluded patients with multiple sclerosis, neuropsychiatric disorders, and other causes of secondary facial pain. The median prescription doses for the primary and repeat GKRS were 80 Gy and $40 \mathrm{~Gy}$, respectively, using a 4-mm collimator. Although there were no strict selection criteria for repeat treatment options, patients who had no initial response to primary GKRS were preferably managed with MVD; however, equal weightage for both treatment arms was given in patients with prior response to primary GKRS. Data on the presence of vascular conflict were obtained from preoperative MRI in GKRS-treated patients and from the operative notes in the MVD-treated patients. Of note, the presence or absence of vascular conflict was not an absolute criterion in patient selection in our institute. Pain outcome was assessed using the 5-point modified Marseille scale at 3-month, 6-month, 1-year, and 2-year intervals when available.

\section{Results}

\section{Systematic Review and Meta-Analysis}

A total of 381 articles and abstracts were identified through electronic database and web search after removing the duplicates. After removing the nonrelevant articles based on the titles and abstracts, a total of 40 articles were reviewed in full text. After careful evaluation, a total of 22 articles were selected for data retrieval and final analysis. $3,5-8,11-16,18-23,25,26,28-30$ Our search failed to identify any study that had directly compared the outcome between repeat GKRS and MVD in patients with prior GKRS failure. Therefore, for purposes of analysis, we grouped the studies into two groups: the first group included studies describing outcome after repeat GKRS $(n=17)$ and the second group included studies describing outcome after MVD $(n=5)$. We also included the findings of our study, which was the only one containing a direct comparison between the two patient cohorts. The PRISMA protocol followed is depicted in Fig. 1.

\section{Study Characteristics}

All the studies were retrospective observational studies except 2, which were prospective. The majority of the studies had patients with idiopathic TN (including patients with typical and atypical pain). Three studies had a small proportion of patients with associated multiple sclerosis. The BNI pain score was the preferred pain outcome measure scale and was described in 11 studies. Other methods of pain outcome assessments were the excellent/good/ fair/poor (EGFP) scale (5 studies) and Marseille scale (3 studies). A comparison of the different scales is shown in Table 1. The total number of patients in the first group (repeat GKRS) was 640 and that in the second group (MVD) was 147. The median follow-up in the first group was 25.8 months (range 10-64.5 months), and that in the second group was 28.5 months (range 21-46 months). The median prescription doses in the first and second GKRS procedures were 77.8 Gy (range 73.4-90 Gy) and 70 Gy (range 40-90 Gy). Most of the studies used nearly the same or a slightly reduced dose in the second GKRS procedure, except in 6 studies (including our study), where the dose was reduced nearly up to half. $3,4,7,14,22,29$ The median intervals between the first and second treatment in the repeat GKRS group and the MVD group were 18 months (range 8-72 months) and 20 months (range 7-45 months), respectively. 


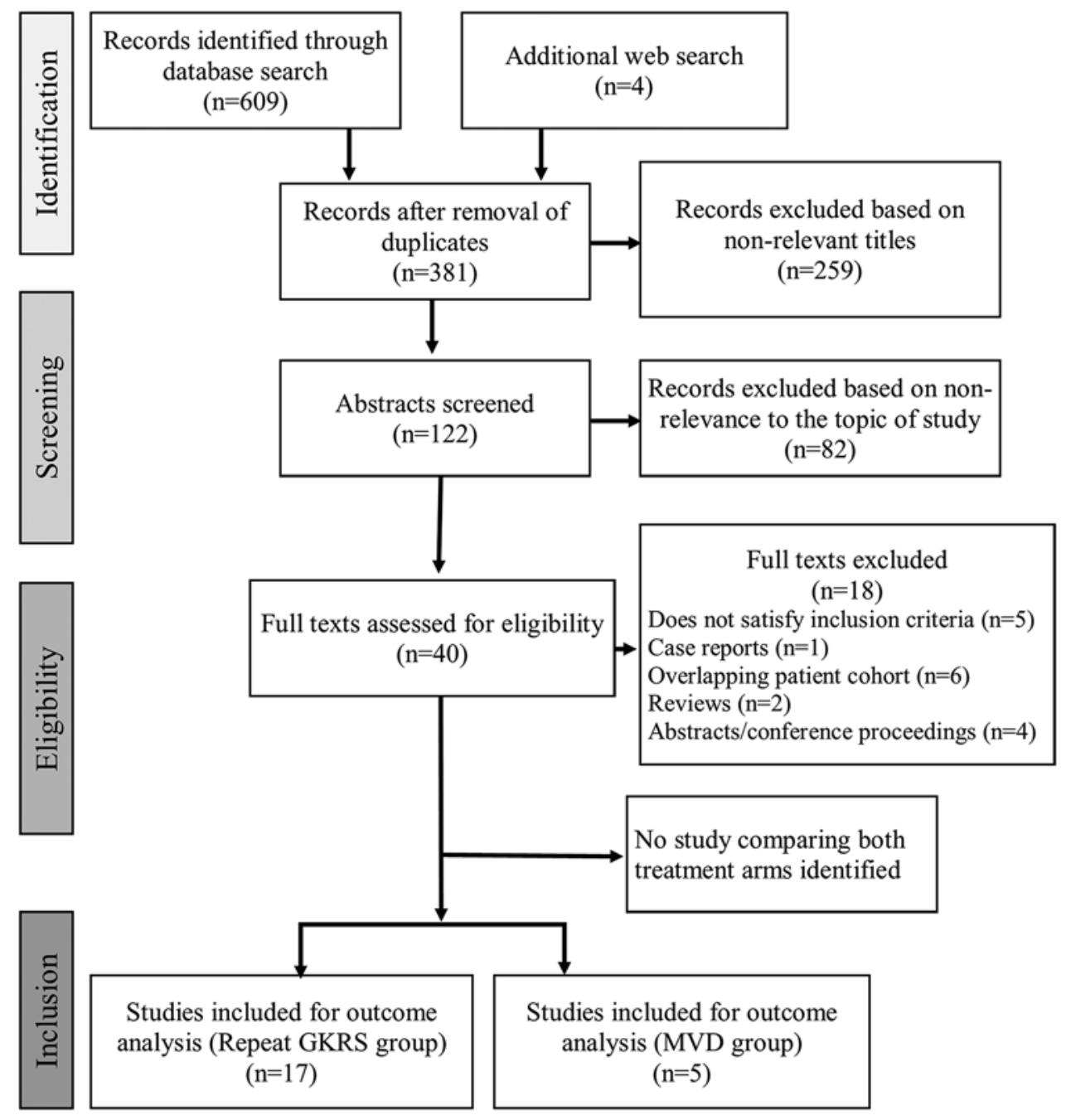

FIG. 1. PRISMA protocol for the included studies.

\section{Outcome Analysis}

Pain Response. The overall estimates of APR 1 year after repeat GKRS and MVD were $83 \%\left(\mathrm{I}^{2}=66 \%, \mathrm{p}<\right.$ $0.01)$ and $88 \%\left(\mathrm{I}^{2}=70 \%, \mathrm{p}<0.01\right)$, respectively (Fig. 2). The overall estimates of CPR 1 year after repeat GKRS and MVD were $46 \%\left(\mathrm{I}^{2}=84 \%, \mathrm{p}<0.01\right)$ and $72 \%\left(\mathrm{I}^{2}=\right.$ $85 \%, \mathrm{p}<0.01)$, respectively. Revised estimates after removing the outlier studies identified by the Baujot plots were not significantly different (Table 2, Supplementary Figs. 1 and 2). A pooled analysis using an independentsample t-test comparing outcomes in both treatment arms showed no significant difference in APR rates $(p=0.49$, $95 \%$ CI -0.16 to -0.08$)$. However, the difference in the CPR rates was statistically significant $(\mathrm{p}=0.02,95 \% \mathrm{CI}$ -0.46 to -0.04 ) (Table 2).

Postoperative Numbness and Retreatment Rates. The overall estimates of postoperative facial numbness (of any degree) after repeat GKRS and MVD are $32 \%\left(\mathrm{I}^{2}=82 \%\right.$, $\mathrm{p}<0.01)$ and $22 \%\left(\mathrm{I}^{2}=46 \%, \mathrm{p}=0.10\right)$, respectively (Fig. 3 ). The rate of bothersome numbness (BNI score III or more) after repeat GKRS was $3 \%$ and that after MVD was
4\%. The retreatment rate was estimated to be $19 \%\left(\mathrm{I}^{2}=\right.$ $61 \%, \mathrm{p}<0.01)$ after repeat GKRS, and that after MVD was $13 \%\left(\mathrm{I}^{2}=73 \%, \mathrm{p}=0.01\right)$. Revised estimates after removing the outlier studies identified by the Baujot plots were not significantly different (Table 2, Supplementary Figs. 1 and 2 ). The comparative analysis did not find any significant difference between repeat GKRS and MVD in the overall rates of postoperative numbness $(\mathrm{p}=0.11,95 \% \mathrm{CI}=-0.02$ to 0.24$)$, bothersome numbness ( $\mathrm{p}=0.31,95 \% \mathrm{CI}-0.07$ to $0.02)$, and retreatment rates $(\mathrm{p}=0.74,95 \% \mathrm{CI}=-0.21$ to 0.27) (Table 2).

\section{Quality of Studies and Publication Bias}

The reporting rate of the APR in the included studies was $100 \%$ for both groups. The CPR reporting rate was $100 \%$ for the MVD group, whereas it was $94 \%$ for the GKRS group. Similarly, the reporting rate of facial numbness was $100 \%$ for both groups, whereas the reporting rate of retreatment was only $67 \%$ in both groups. Thus, overall, the reporting rate of all the outcomes was consistent among the studies except for retreatment rates. Heteroge- 


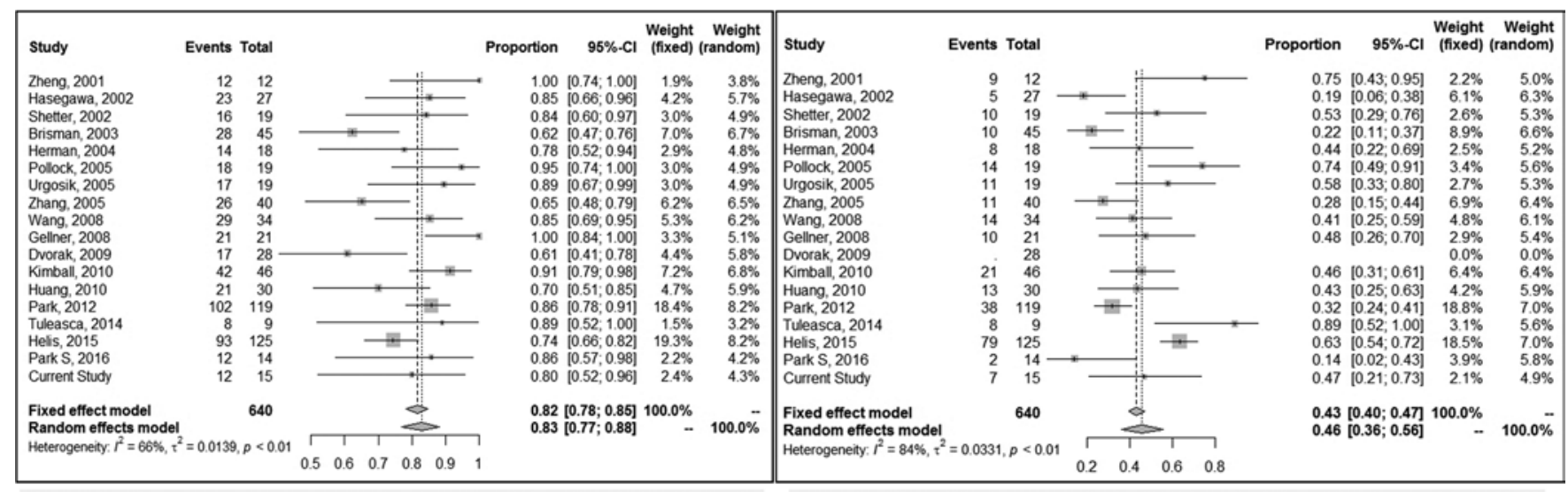

APR-Repeat GKRS

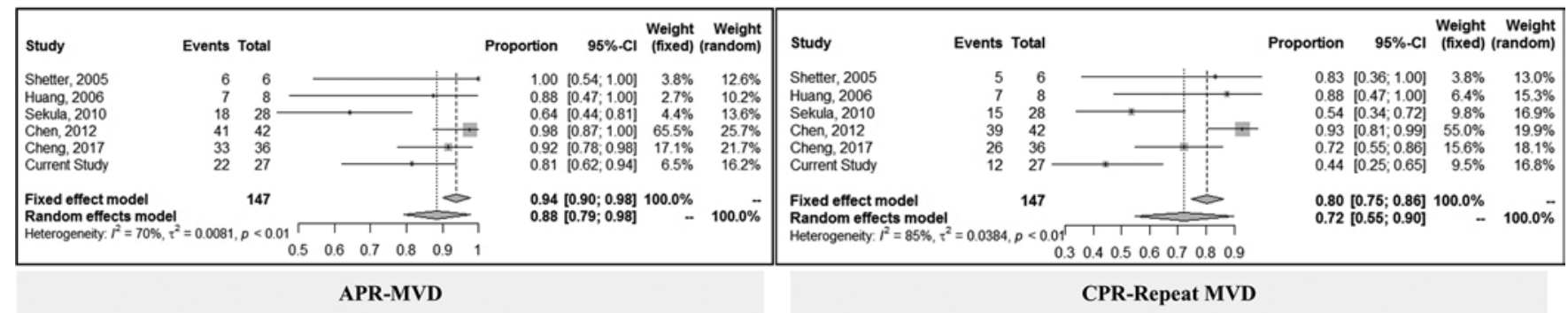

FIG. 2. Forest plots of overall estimates of APR and CPR after repeat GKRS and MVD.

neity was an expected concern among the studies, which led us to include slight variations in the statistical methods. From the quality scoring using the MOOSE checklist, it was evident that the included studies satisfy all the quality criteria except for one (identification of prognostic factors and important confounders). The funnel plots with Egger's test did not identify any significant publication bias for reporting of primary outcomes, except for APR in the MVD group (Fig. 4).

\section{Assessment of Treatment Bias}

We systematically evaluated the presence of bias in selecting patients for a particular treatment arm. It is plausible that patients who are old or unfit for surgery are naturally selected for GKRS, and similarly patients who did not have a response after initial GKRS are selected for MVD. However, the patient cohorts in the included studies were quite heterogeneous, and the majority did not report any specific selection criteria. All the studies in the MVD group included patients irrespective of prior response to GKRS. In the repeat GKRS group, half the studies had no clear mention of their selection criteria, two included patients who were not surgical candidates, and only three studies had categorically included patients who had a prior response. Therefore, it is evident that a treatment bias existed in a few studies, but the bias was not applicable to all.

\section{Our Institutional Study}

A total of 198 patients with idiopathic TN were treated with primary GKRS in the study period. Of these, 42 patients required repeat treatment (repeat GKRS 15; MVD 27) due to failure or relapse and were included in our analysis. The mean age of the patients was 59 years, and the male to female ratio was 1:2. Data on the presence of vascular conflict could be obtained in 9 of the GKRS and

TABLE 2. Summary of overall estimates of outcome rates

\begin{tabular}{cccc}
\hline Outcome & Repeat GKRS $(95 \%$ Cl of proportions) & MVD $(95 \%$ Cl of proportions) & $p$ Value $(95 \%$ Cl) \\
\hline Primary & & & \\
\hline APR & $83 \%(0.77 ; 0.88)$ & $88 \%(0.79 ; 0.98)[87.5 \%]$ & $0.49(-0.16 ; 0.08)$ \\
\hline CPR & $46 \%(0.36 ; 0.56)[45.8 \%]$ & $72 \%(0.55 ; 0.90)$ & $0.02(-0.46 ;-0.04)$ \\
\hline Secondary & & & \\
\hline Numbness & $32 \%(0.24 ; 0.40)[31.7 \%]$ & $22 \%(0.12 ; 0.31)[21.8]$ & $0.25(-8.1 ; 29.5)$ \\
\hline Bothersome numbness & $3 \%(0.01 ; 0.05)$ & $4 \%(0.00 ; 0.11)$ & $0.31(-0.07 ; 0.02)$ \\
\hline Retreatment & $19 \%(0.13 ; 0.25)[19 \%]$ & $22 \%(0.12 ; 0.31)[21.5]$ & $0.74(-0.21 ; 0.27)$ \\
\hline
\end{tabular}

Values within brackets are pooled estimates without outlier studies. 
Patra et al.

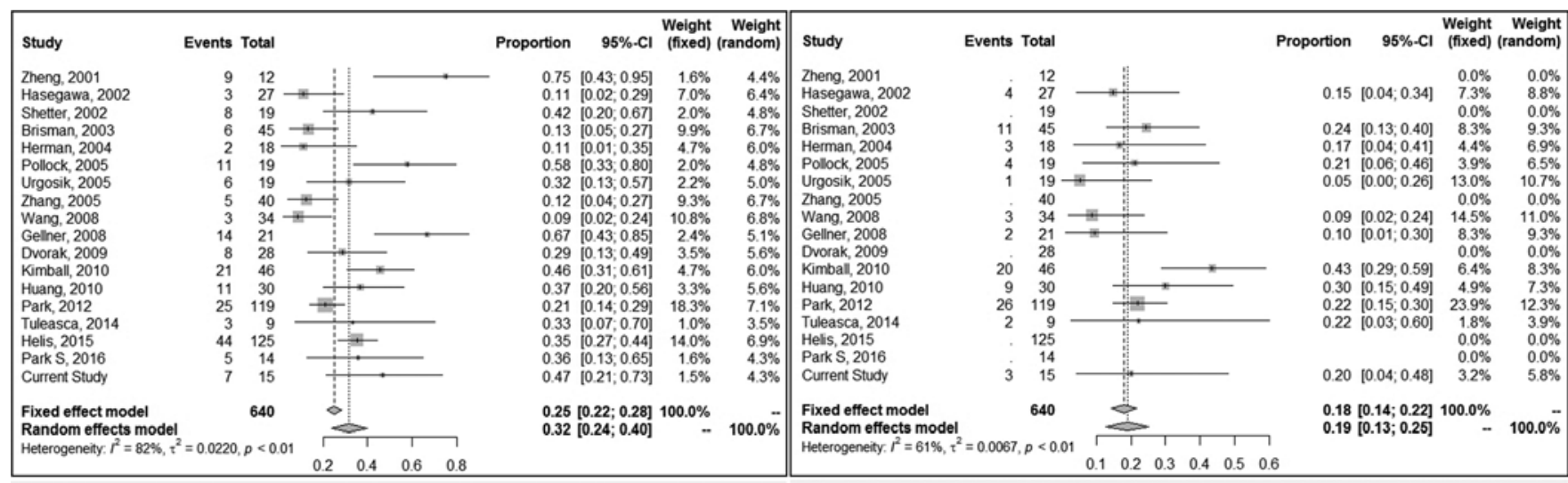

Facial Numbness-Repeat GKRS

Retreatment-Repeat GKRS

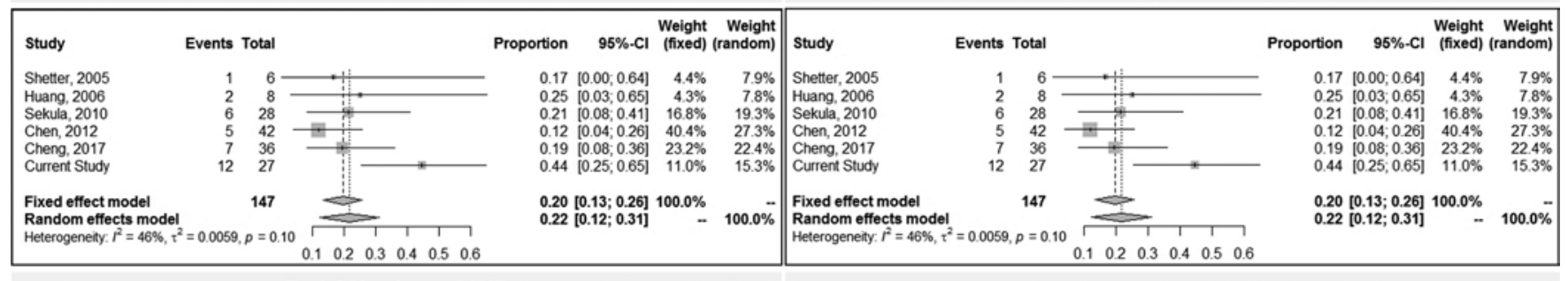

Facial Numbness-MVD

Retreatment-MVD

FIG. 3. Forest plots of overall estimates of facial numbness and retreatment rate after repeat GKRS and MVD.
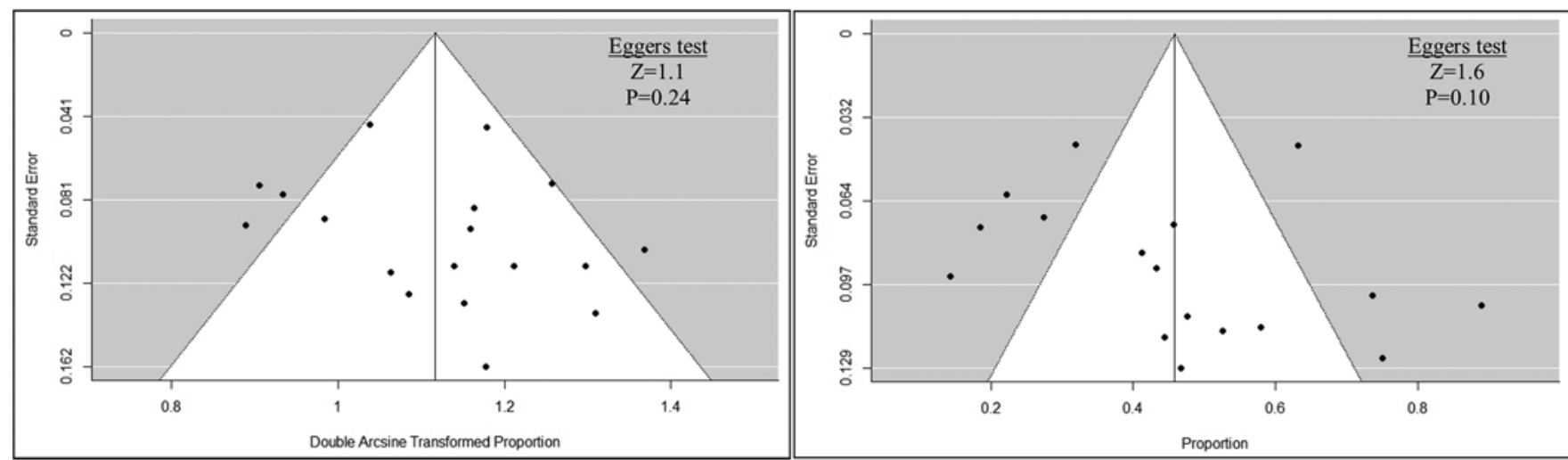

APR-Repeat GKRS

CPR-Repeat GKRS

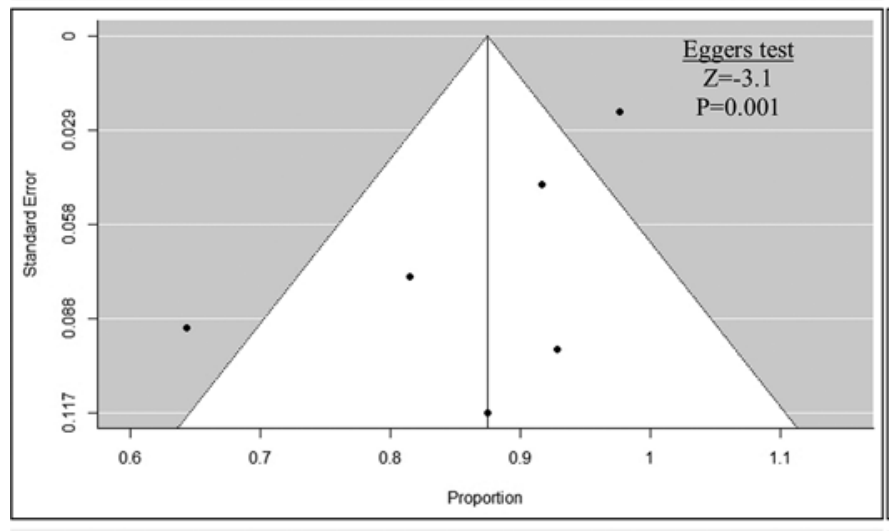

APR-MVD

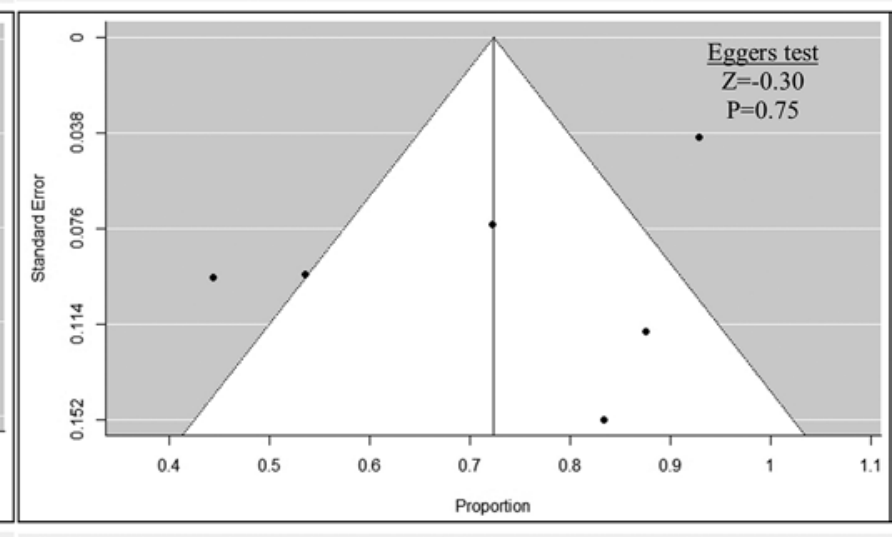

CPR-MVD

FIG. 4. Funnel plot for publication bias in APR and CPR outcome reporting after repeat GKRS and MVD. 


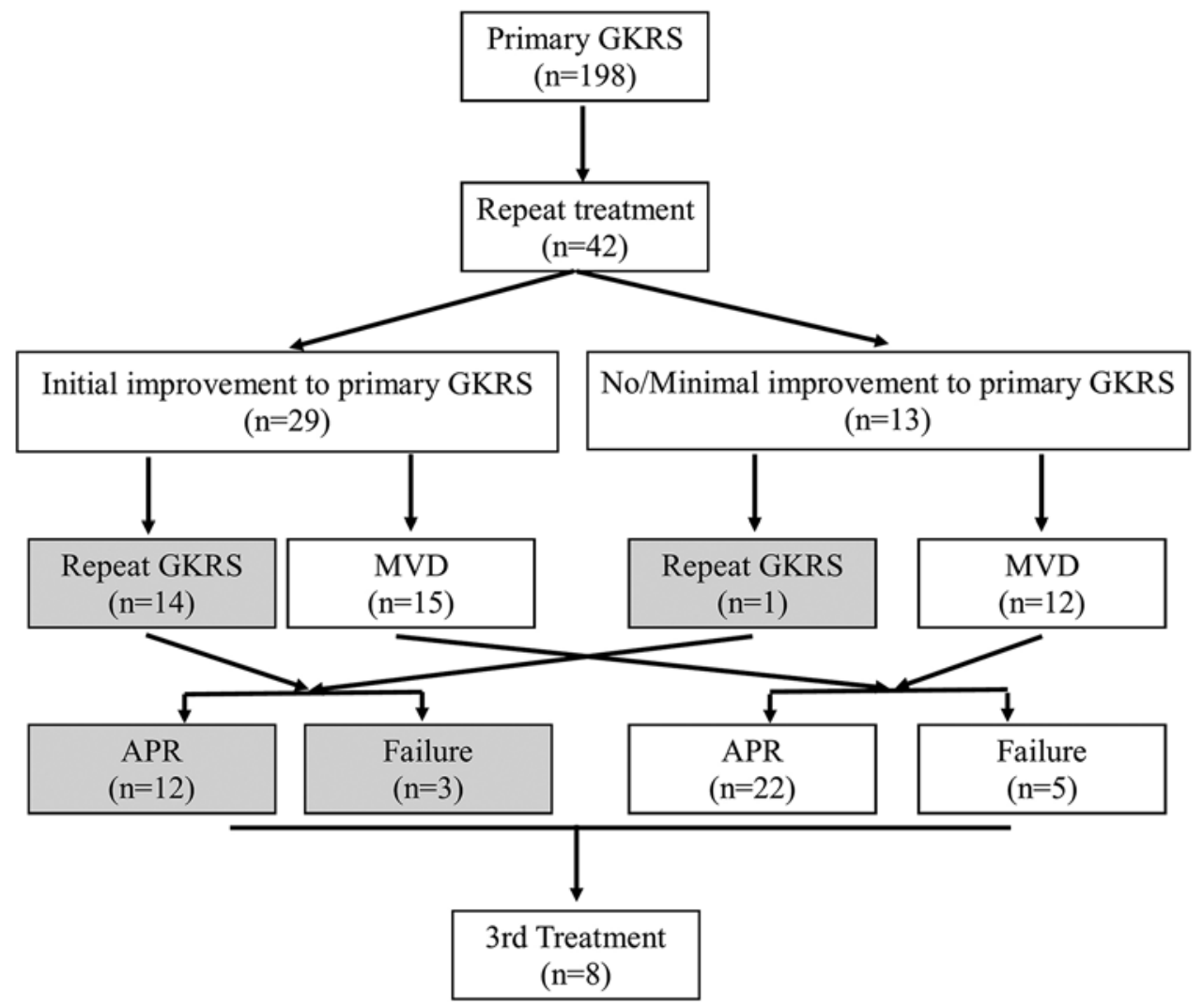

FIG. 5. Summary of treatment protocol followed in our institute.

21 of the MVD patients. Vascular compression was evident in 3 and 16 patients in the GKRS and MVD groups, respectively. A treatment and outcome tree is depicted in Fig. 5. The APR rates after retreatment were as follows: at 3 months for GKRS and MVD, 93\% and 96\% ( $\mathrm{p}=1.0)$; at 6 months for GKRS and MVD, 87\% and 89\% (p = 1.0); at 1 year for GKRS and MVD, 80\% and 81\% ( $\mathrm{p}=1.0$ ); and at 2 years for GKRS and MVD, 67\% and 74\% ( $p=0.6)$, respectively. The CPR rates after retreatment were as follows: at 3 months for GKRS and MVD, 33\% and 67\% (p = $0.055)$; at 6 months for GKRS and MVD, 33\% and 44\% (p $=0.53)$; at 1 year for GKRS and MVD, 47\% and 44\% $(\mathrm{p}=$ 1.0); and at 2 years for GKRS and MVD, $47 \%$ and $44 \%$ (p = 1.0). Among the patients who had a prior response to initial GKRS $(n=29), 78.5 \%$ from the GKRS group and $86.6 \%$ from the MVD group achieved APR ( $p=0.65)$, and $50 \%$ from the GKRS group and $46.6 \%$ from the MVD group achieved CPR $(\mathrm{p}=1.0)$. In the group of patients who did not have a prior response to initial GKRS $(n=13)$, only one patient underwent repeat GKRS, and this patient achieved APR but not CPR at 1 year. Among the other patients who underwent MVD, APR and CPR were achieved in $75 \%$ and $42 \%$ of patients, respectively. There was no difference in the rate of postoperative facial numbness at 1 year (GKRS group, 46.6\%; MVD group, 44.4\% [p = 1.0]). Eight patients required subsequent treatment (3 from the repeat GKRS group and 5 from the MVD group [p=1.0]). The probability of APR and CPR at different time intervals was depicted using Kaplan-Meier estimates (Fig. 6).

\section{Discussion}

\section{Role of Repeat GKRS in TN}

GKRS is an attractive option as a repeat treatment because of its noninvasive nature and its feasibility in older patients and in patients with multiple comorbidities that preclude surgical treatment. However, the effect of GKRS is often delayed, and most of the series have reported the optimal effect to occur from 1 to 11 weeks after the procedure., $711,13,16,20,22$ Several studies report a comparable or even better pain response with repeat GKRS compared to primary GKRS. However, the major concern about repeat GKRS treatment is its radiotoxicity added to an already irradiated nerve. ${ }^{25}$ There have been multiple conflicting reports on the ideal and effective cumulative dose that is safe and effective. It has been shown that there is a doseresponse relationship between the cumulative radiation dose and the pain response rate along with incidence of trigeminal dysfunction. ${ }^{7}$ A few studies recommend a high cumulative dose (>150 Gy), which is tolerable and effective, ${ }^{20,26}$ but a low-dose strategy ( $<130 \mathrm{~Gy}$ ) has also been shown equally effective..$^{3,729}$ Hasegawa et al. compared the results of low-dose and high-dose radiation and found no difference in the overall outcome in their patients. ${ }^{11}$ In contrast, a recent meta-analysis has shown poor pain control with a low cumulative dose $\left(<140-150\right.$ Gy). ${ }^{19}$ Because individual variation in radiation sensitivity might have a role in the final outcome, a superselective strategy to identify suitable patients for repeat treatment has been recently put 

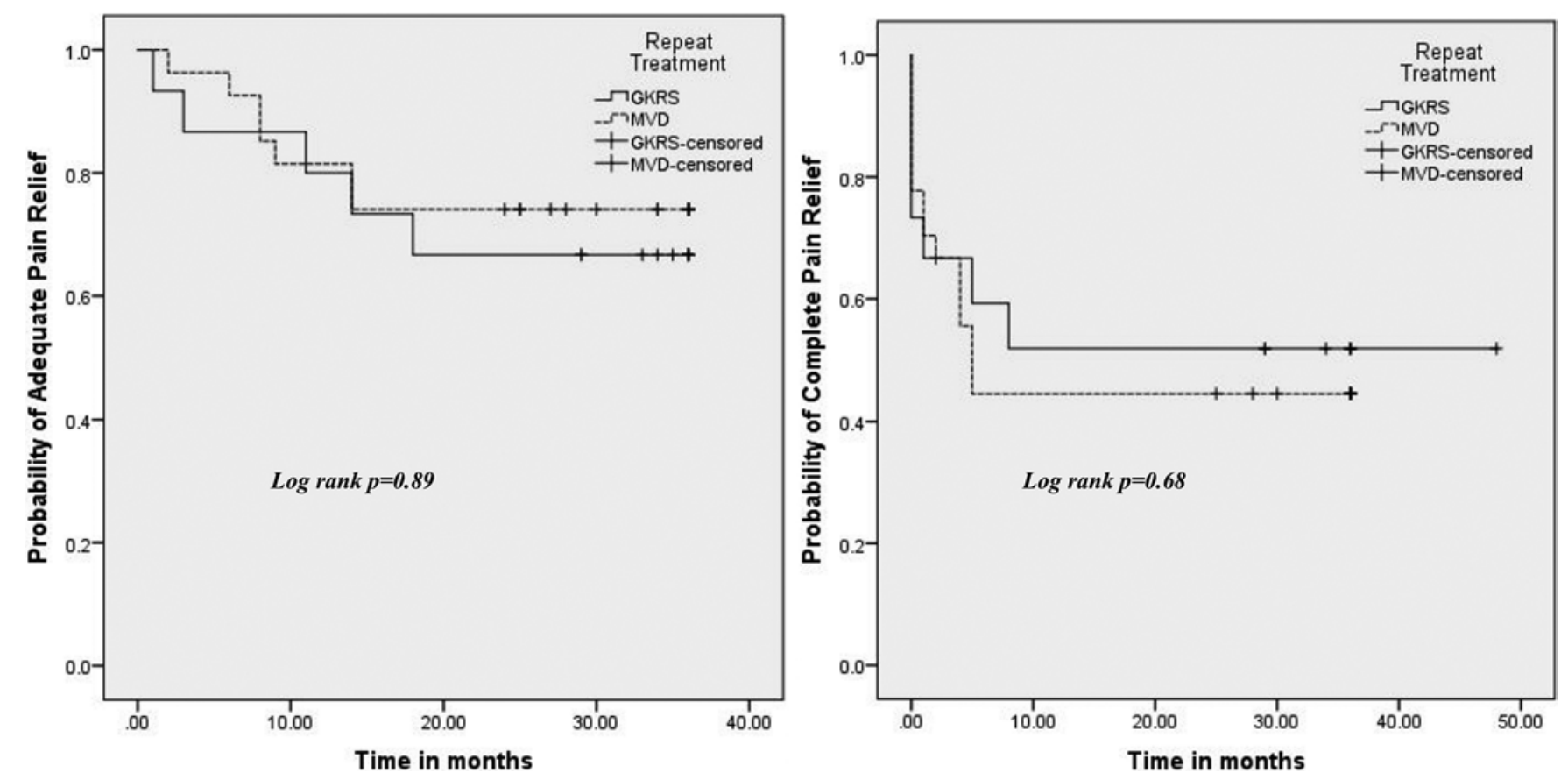

FIG. 6. Kaplan-Meier curves showing the probability of APR and CPR over time after repeat GKRS and MVD in our institutional study.

forth. In this regard, Tuleasca et al. reviewed 20 studies with 626 patients with recurrent $\mathrm{TN}$ who received repeat radiosurgery. ${ }^{25}$ The review identified some of the important positive predictors for initial pain relief, including 1) initial CPR after a prior GKRS, 2) longer period of pain relief after first GKRS, and 3) new trigeminal deficits. The negative predictors included 1) no pain relief after prior GKRS and 2) a prior surgical procedure. This fact has been further validated by other authors who emphasized a higher probability of pain response in patients with prior history of a positive response to GKRS. ${ }^{13,28}$

\section{Role of MVD After Failure of GKRS in TN}

MVD has been regarded as the gold-standard treatment for TN. Two recently published meta-analyses have established the superiority of MVD as a primary treatment over GKRS due to higher initial pain-free rates and long-term pain-free rates. ${ }^{9,17}$ However, the role of MVD after failure of other procedures has been less clearly established than its role as a primary procedure. The primary mechanism by which MVD produces pain relief in patients with vascular compression (reversal of physiological changes produced by demyelination) is altered with prior ablative procedures, including GKRS. Although not directly proven, such lesioning procedures produce a change in the electrophysiological properties of the nerve, making the effect of subsequent surgical decompression unreliable. Barba and Alksne, in an early report, demonstrated an inferior outcome in MVD-treated patients who had undergone prior ablative procedures compared to those who underwent MVD as the primary surgery. ${ }^{1}$ However, recently, Chen has argued that prior therapy does not adversely affect pain outcome after MVD.5 Still, most clinicians would agree that the response of MVD as a salvage procedure is not as good as it is for a primary procedure. The primary concern for MVD in a previously irradiated patient is the possible alteration of the nerve-vessel anatomy with high incidence of adhesions, scarring, etc., which may make the surgical decompression more challenging. However, multiple reports have documented successful attempts of MVD in patients who have undergone prior GKRS, with no difficulty with vascular dissection. ${ }^{15,23}$ Sekula et al. have provided a more comprehensive and detailed report on operative findings in this group of patients. ${ }^{21}$ In their 29 patients, they found trigeminal nerve atrophy in 14 (48.2\%), adhesion of the trigeminal nerve in $6(20.6 \%)$, and thickened arachnoid in 1 (3.4\%). Although they have reported no difficulty during dissection in most of their cases, they had to abandon one procedure due to gross adhesion of the nerve to the vessel. In our series, we did not encounter any difficulty in dissection during the surgery. The current perspective is that MVD is a feasible and effective modality of treatment without any added morbidity due to the prior radiation procedure.

\section{Repeat GKRS Versus MVD in the Setting of Prior GKRS Failure}

Multiple studies have reported the effectiveness of repeat GKRS or MVD in patients with prior failure of GKRS, but we did not encounter any study that has directly compared the two interventions. Therefore, the current meta-analysis focused on the pooled estimates of outcome measures in the two mutually exclusive groups of patients. In addition, we have compared the two interventions in our own patient series to get a preliminary estimate on a direct head-to-head comparison. Indirect comparison from our 
meta-analysis shows that MVD provides a significantly better rate of CPR compared with repeat GKRS (73\% vs $45 \%$ ); however, both treatment modalities provide a reasonable rate of APR (87\% and $80 \%$ in MVD and repeat GKRS, respectively). Similarly, the only statistically significant difference in our series among the two groups was the CPR rates at 3 months, with almost double the rate of complete relief with MVD compared to GKRS; however, the difference was nullified over time. The possible explanation for this difference would be the almost immediate and complete response with surgical decompression of the nerve compared with GKRS, which usually has a long latency period for optimal response. Both the meta-analysis of studies from literature and our own institutional study did not identify any significant difference in the rates of postoperative facial numbness or retreatment between these two treatment arms. Although repeat GKRS has a higher incidence of new facial numbness than MVD, most cases of numbness are not bothersome and therefore are less likely to affect the quality of life.

MVD has been regarded as the gold-standard treatment for TN, especially in the setting of prior failure with GKRS. Similarly, repeat GKRS, being noninvasive, has a definite role in patients with a prior good response to GKRS. Although a treatment bias cannot be excluded, the majority of the included studies did not report any treatment preference for patients with or without prior response. As evident from the analysis, MVD is an attractive option as it provides a better rate of CPR. However, considering that it has a similar overall APR and is a noninvasive therapeutic modality, repeat GKRS is still a comparable option. Although solid evidence is lacking, a general guideline that is helpful in selecting patients would be to use repeat GKRS in patients with prior response to GKRS, in the elderly, and in patients with comorbidities and to use MVD in all other patients, especially those who are young.

\section{Study Limitations}

The most important limitation of the current metaanalysis is the absence of direct comparison groups, except our own series. Although our series provides a preliminary comparison between the two interventions, the lack of randomization and the retrospective nature of the study limit the validity of the results. Pertaining to the included studies, heterogeneity and publication bias were important issues, but they were largely unavoidable. Although most of the studies have nearly comparable and objective criteria for outcome measures, minor errors in reporting and data extraction cannot be ruled out. A minority of the patients in the included studies had failure/ recurrence but did not undergo a retreatment for various reasons. This attrition might have had a minor influence on the analysis of retreatment rate. In addition, our analysis did not extend to subgroups (e.g., patients with partial vs no response to initial GKRS) to identify certain groups of patients who might benefit from select treatment methods. Of note, $20 \%-40 \%$ of the patients in the included studies had one or more other treatment modalities prior to the initial GKRS, which might have some influence on the pain response after repeat GKRS or MVD.

\section{Conclusions}

Repeat GKRS and MVD are both valid treatment options for patients after failure of prior GKRS. The current meta-analysis failed to identify any superiority of one treatment over the other, showing comparable outcomes in terms of APR, postoperative facial numbness, and retreatment rates. However, MVD provided a better chance of CPR than repeat GKRS. A large-scale randomization of patients with subgroup analysis may help to identify select patients who may benefit from any particular modality of retreatment.

\section{Acknowledgments}

We thank Ms. Gloria Caldito, associate professor in the Department of Statistics and Research, Louisiana State University, Shreveport, for assistance with the statistical analysis.

\section{References}

1. Barba D, Alksne JF: Success of microvascular decompression with and without prior surgical therapy for trigeminal neuralgia. J Neurosurg 60:104-107, 1984

2. Brisman R: Microvascular decompression vs. gamma knife radiosurgery for typical trigeminal neuralgia: preliminary findings. Stereotact Funct Neurosurg 85:94-98, 2007

3. Brisman R: Repeat gamma knife radiosurgery for trigeminal neuralgia. Stereotact Funct Neurosurg 81:43-49, 2003

4. Chen AY, Li Q, Wang Y, Xu KY, Pappas C: Outcome of repeat stereotactic radiosurgery for trigeminal neuralgia by linear accelerator. Int J Radiat Oncol Biol Phys 99:E645E645, 2017

5. Chen JCT: Microvascular decompression for trigeminal neuralgia in patients with and without prior stereotactic radiosurgery. World Neurosurg 78:149-154, 2012

6. Cheng J, Liu W, Hui X, Lei D, Zhang H: Microvascular decompression for trigeminal neuralgia in patients with failed gamma knife surgery: analysis of efficacy and safety. Clin Neurol Neurosurg 161:88-92, 2017

7. Dvorak T, Finn A, Price LL, Mignano JE, Fitzek MM, Wu $\mathrm{JK}$, et al: Retreatment of trigeminal neuralgia with Gamma Knife radiosurgery: is there an appropriate cumulative dose? Clinical article. J Neurosurg 111:359-364, 2009

8. Gellner V, Kurschel S, Kreil W, Holl EM, Ofner-Kopeinig P, Unger F: Recurrent trigeminal neuralgia: long term outcome of repeat gamma knife radiosurgery. J Neurol Neurosurg Psychiatry 79:1405-1407, 2008

9. Gubian A, Rosahl SK: Meta-analysis on safety and efficacy of microsurgical and radiosurgical treatment of trigeminal neuralgia. World Neurosurg 103:757-767, 2017

10. Guo S, Chao ST, Reuther AM, Barnett GH, Suh JH: Review of the treatment of trigeminal neuralgia with gamma knife radiosurgery. Stereotact Funct Neurosurg 86:135-146, 2008

11. Hasegawa T, Kondziolka D, Spiro R, Flickinger JC, Lunsford LD: Repeat radiosurgery for refractory trigeminal neuralgia. Neurosurgery 50:494-502, 2002

12. Helis CA, Lucas JT Jr, Bourland JD, Chan MD, Tatter SB, Laxton AW: Repeat radiosurgery for trigeminal neuralgia. Neurosurgery 77:755-761, 2015

13. Herman JM, Petit JH, Amin P, Kwok Y, Dutta PR, Chin LS: Repeat gamma knife radiosurgery for refractory or recurrent trigeminal neuralgia: treatment outcomes and quality-of-life assessment. Int J Radiat Oncol Biol Phys 59:112-116, 2004

14. Huang CF, Chiou SY, Wu MF, Tu HT, Liu WS: Gamma Knife surgery for recurrent or residual trigeminal neuralgia after a failed initial procedure. J Neurosurg 113 Suppl:172-177, 2010 
15. Huang CF, Chuang JC, Tu HT, Chou MC: Microsurgical outcomes after failed repeated Gamma Knife surgery for refractory trigeminal neuralgia. J Neurosurg 105 Suppl:117-119, 2006

16. Kimball BY, Sorenson JM, Cunningham D: Repeat Gamma Knife surgery for trigeminal neuralgia: long-term results. J Neurosurg 113 Suppl:178-183, 2010

17. Mendelson ZS, Velagala JR, Kohli G, Heir GM, Mammis A, Liu JK: Pain-free outcomes and durability of surgical intervention for trigeminal neuralgia: a comparison of Gamma Knife and microvascular decompression. World Neurosurg 112:e732-e746, 2018

18. Park KJ, Kondziolka D, Berkowitz O, Kano H, Novotny J Jr, Niranjan A, et al: Repeat gamma knife radiosurgery for trigeminal neuralgia. Neurosurgery 70:295-305, 2012

19. Park SC, Kwon DH, Lee DH, Lee JK: Repeat Gamma-Knife radiosurgery for refractory or recurrent trigeminal neuralgia with consideration about the optimal second dose. World Neurosurg 86:371-383, 2016

20. Pollock BE, Foote RL, Link MJ, Stafford SL, Brown PD, Schomberg PJ: Repeat radiosurgery for idiopathic trigeminal neuralgia. Int J Radiat Oncol Biol Phys 61:192-195, 2005

21. Sekula RF Jr, Frederickson AM, Jannetta PJ, Bhatia S, Quigley MR: Microvascular decompression after failed Gamma Knife surgery for trigeminal neuralgia: a safe and effective rescue therapy? J Neurosurg 113:45-52, 2010

22. Shetter AG, Rogers CL, Ponce F, Fiedler JA, Smith K, Speiser BL: Gamma knife radiosurgery for recurrent trigeminal neuralgia. J Neurosurg 97 (5 Suppl):536-538, 2002

23. Shetter AG, Zabramski JM, Speiser BL: Microvascular decompression after gamma knife surgery for trigeminal neuralgia: intraoperative findings and treatment outcomes. J Neurosurg 102 Suppl:259-261, 2005

24. Tawk RG, Duffy-Fronckowiak M, Scott BE, Alberico RA, Diaz AZ, Podgorsak MB, et al: Stereotactic gamma knife surgery for trigeminal neuralgia: detailed analysis of treatment response. J Neurosurg 102:442-449, 2005

25. Tuleasca C, Carron R, Resseguier N, Donnet A, Roussel P, Gaudart J, et al: Repeat Gamma Knife surgery for recurrent trigeminal neuralgia: long-term outcomes and systematic review. J Neurosurg 121 Suppl:210-221, 2014

26. Urgosik D, Liscak R, Novotny J Jr, Vymazal J, Vladyka V: Treatment of essential trigeminal neuralgia with gamma knife surgery. J Neurosurg 102 Suppl:29-33, 2005

27. Wang DD, Raygor KP, Cage TA, Ward MM, Westcott S, Barbaro NM, et al: Prospective comparison of long-term pain relief rates after first-time microvascular decompression and stereotactic radiosurgery for trigeminal neuralgia. J Neurosurg 128:68-77, 2018

28. Wang L, Zhao ZW, Qin HZ, Li WT, Zhang H, Zong JH, et al: Repeat gamma knife radiosurgery for recurrent or refractory trigeminal neuralgia. Neurol India 56:36-41, 2008

29. Zhang P, Brisman R, Choi J, Li X: Where to locate the isocenter? The treatment strategy for repeat trigeminal neuralgia radiosurgery. Int J Radiat Oncol Biol Phys 62:38-43, 2005

30. Zheng LG, Xu DS, Kang CS, Zhang ZY, Li YH, Zhang YP, et al: Stereotactic radiosurgery for primary trigeminal neuralgia using the Leksell Gamma unit. Stereotact Funct Neurosurg 76:29-35, 2001

\section{Disclosures}

The authors report no conflict of interest concerning the materials or methods used in this study or the findings specified in this paper.

\section{Author Contributions}

Conception and design: Patra. Acquisition of data: Patra, Savardekar, Dossani, Narayan. Analysis and interpretation of data: Patra, Savardekar, Mohammed. Drafting the article: Patra. Critically revising the article: all authors. Reviewed submitted version of manuscript: all authors. Approved the final version of the manuscript on behalf of all authors: Nanda. Statistical analysis: Patra. Administrative/technical/material support: Nanda. Study supervision: Nanda.

\section{Supplemental Information \\ Online-Only Content}

Supplemental material is available with the online version of the article.

Supplementary Figs. 1 and 2. https://thejns.org/doi/suppl/ 10.3171/2018.5.JNS18583.

\section{Previous Presentations}

A portion of the present study was published in abstract form for an oral presentation at the CNS 2017 Annual Meeting, Boston, Massachusetts, October 7-11, 2017.

\section{Correspondence}

Anil Nanda: LSU Health Shreveport, Shreveport, LA. ananda@ lsuhsc.edu. 